\title{
A Velha Sexualidade nos Idosos de Hoje: Quando Surge a Demência
}

\section{Sexuality in the Elderly of Today: When Does the Dementia}

\author{
Diana Devesa*, Helena Cruz*, José Augusto Simões**
}

\section{Pontos-chave:}

Como adultos, também os indivíduos demenciados sentem a necessidade de expressar a sua sexualidade.

A gestão do comportamento sexual na época de aparecimento da demência, especialmente nos institucionalizados, implica decisões de ordem ética moral, pelos profissionais que com eles lidam.

\section{Resumo}

O século XX ficou marcado por alterações na estrutura populacional. Este fenómeno está associado a um declínio da fecundidade, que leva a uma diminuição da percentagem de jovens e, consequentemente, a um aumento da percentagem de idosos.

Esta importância crescente dos idosos no panorama demográfico faz com que as questões que dizem respeito a esta faixa etária assumam uma importância cada vez maior. Assim, a abordagem psicológica desta etapa da vida torna-se fundamental, justificando-se, portanto, o estudo de temáticas que possam contribuir para o bem-estar do idoso, como é o caso da sexualidade.

Esta é uma área considerada tabu na actual sociedade, principalmente quando associada ao idoso. Contudo, o facto de o indivíduo ter uma esperança de vida cada vez maior, alarga também todo um conjunto dos papéis sócio-afectivos que este desempenhou ao longo da sua vida, incluindo a sua sexualidade.

Uma das variáveis mais importantes associadas com a actividade sexual na terceira idade é o estado de saúde mental dos indivíduos. Sendo a demência uma doença associada à terceira idade, com o aumento da longevidade assiste-se cada vez mais, à multiplicação de casos desta patologia. Como adultos, também os indivíduos demenciados sentem a necessidade de expressar

\begin{abstract}
Changes in population structure marked the 20th century. This phenomenon, associated to a decline in fertility, lead to a decrease in youth population and, consequently, a higher proportion of elderly.
\end{abstract}

This escalating significance, in the panorama of the elderly population, causes the issues that concern this age group to assume an increasing importance. The psychological approach to this stage of life is also fundamental; justifying therefore, the study of subjects that may contribute to the welfare of the elderly, such as sexuality.

This is an area, still considered taboo in today's society, especially when associated to aged people. However, due to the fact that life expectancy increased, a whole set of social-emotional roles played by an individual throughout one's life also extended, including their sexuality.

One of the most important variables associated with sexual activity, in old age, is the mental health condition. Being dementia a disease associated to old age, due to the increasing of longevity, we are witnessing increasingly to the plethora of cases of this disease. As adults, those with dementia also feel the need to express their sexuality, which brings, to the light of society, problems with the management of their sexual behavior, especially when within an institution. This leads to the need of making decisions based on ethical and moral order,

\footnotetext{
*Alunas do Mestrado em Gerontologia da Universidade de Aveiro

*** Professor no Mestrado em Gerontologia da Universidade de Aveiro no ano letivo 2010-2011.
} 
a sua sexualidade, o que traz, à luz da sociedade problemas ligados com a gestão do seu comportamento sexual especialmente dentro de uma instituição, o que leva à necessidade da tomada de decisões de ordem ético moral, por parte dos profissionais que lidam com estes utentes. Sendo assim necessário um maior foco de atenção pelo tema.

Palavra-chave: idoso, sexualidade, demência.

\section{Introdução}

Durante o século XX, principalmente na segunda metade, ocorreu uma grande mudança no perfil etário das populações em relação ao aumento da longevidade.

Vivemos num mundo em envelhecimento, sendo que em 2002, 629 milhões de pessoas tinham 60 ou mais anos. Estima-se que este grupo etário será composto por 2 biliões de pessoas até ao ano de 2050. Esta mudança na estrutura etária da população mundial é considerada como um dos maiores desafios do século XXI, trazendo consigo novos desafios sociais, económicos, políticos e científicos ${ }^{1}$

O crescente envelhecimento populacional, resultante do aumento da qualidade de vida e consequente engrandecimento da longevidade, assim como a diminuição da taxa de natalidade, tem vindo a direccionar estudos no sentido de melhorar a resposta dos vários profissionais que integram o conjunto de intervenientes que diariamente lidam com a terceira idade, uma vez que o envelhecer deixou de constituir um problema meramente psicológico, pessoal e familiar para se tornar num problema social. ${ }^{2}$

Juntamente com os novos desafios, esta rápida e intensa transição demográfica trouxe à superfície algumas questões que necessitam de uma ampla reflexão, e que têm como propósito atender às diversas demandas desta faixa etária.

Face a estes dados, levanta-se uma importante questão relacionada com as enfermidades e distúrbios que surgem com a idade, sendo que destas se destacam as alterações psicofisiológicas que, de certa forma, condicionarão a sexualidade e a capacidade sexual, que ao contrário do que se possa pensar existe e está presente nesta faixa etária assim como outras necessidades básicas. by professionals who deal with these users. Therefore, it is a major focus of attention by the theme.

Key-words: elderly, sexuality, dementia.

\section{Sexualidade no Idoso}

A Organização Mundial de Saúde define sexualidade como sendo "uma energia que dá motivação para encontrar amor, contacto, ternura e intimidade; integra-se no modo como nos sentimos, movemos, tocamos e somos tocados; é ser-se sensual e ser-se sexual. A sexualidade influencia pensamentos e, por isso, influencia também a saúde física e mental." ${ }^{3}$

A sexualidade, bem como todos os sentimentos e sensações que the estão associados, sempre fizeram parte da história da humanidade quer de uma forma mais subtil ou de uma forma mais clara e explícita. Ao longo dos tempos a expressão e vivência da sexualidade tem estado associada a conceitos de pecado e a ideias préconcebidas, resultantes da rigidez ético moral da Igreja Católica vigente na nossa sociedade. ${ }^{2}$

A sexualidade faz parte da vida dos seres humanos e está presente em todas as fases do desenvolvimento do homem, e a sua função sexual permanece durante toda a vida incluindo na terceira idade. ${ }^{4}$

O comportamento sexual é definido por vários princípios: cultura, religião, educação e estes valores influenciam intensamente o desenvolvimento sexual, determinando a maneira como iremos vivenciá-la e lidar com ela por toda a vida. Segundo alguns autores, quando se trata da terceira idade, o contexto social em que se insere o idoso tem grande influência. ${ }^{4}$

A sexualidade na fase tardia da vida permanece um tabu e muitas vezes a mera sugestão de que a população idosa pode permanecer sexualmente activa despoleta, aos mais novos, uma resposta emocional de descrença, desconforto e até divertimento. Em contraste, os mais velhos parecem encarar o sexo como uma actividade natural nesta fase das suas vidas. ${ }^{5}$ 
Para Azevedo, tanto o homem como a mulher continuam a apreciar as relações sexuais durante a velhice, no entanto as alterações que ocorrem na mulher, como a secura da vagina e a diminuição no tempo de erecção do homem, podem prejudicar o prazer sexual, sendo que a boa adaptação sexual determinará o prazer. ${ }^{6}$

Ao contrário daquilo que se pensa, os idosos não são seres assexuados, podendo mesmo expressar e vivenciar a sua sexualidade até idades muito avançadas. Este facto pode ser comprovado através da análise dos estudos realizados por Pfeiffer na década de 60, de onde veio a concluir que o desejo e os interesses sexuais prevalecem na velhice da seguinte forma: entre os 60 e os 65 anos, estão presentes em 60\% das mulheres e 80\% dos homens; nos indivíduos com idade superior a 70 anos verificam-se em $26 \%$ das mulheres e em $61 \%$ dos homens. Também os comportamentos sexuais com envolvimento do coito foram contemplados e concluíram que acontecem em $49 \%$ das mulheres e $69 \%$ dos homens entre os 60 e os 65 anos e em 22\% das mulheres e $30 \%$ dos homens com mais de 70 anos. $^{7}$

Como verificado pelos anteriores descritos, a sexualidade na terceira idade é um facto real encarado pela população idosa como um fenómeno normal, pertencente e integrante das suas vidas. No entanto, apesar desta realidade, a sexualidade nesta faixa etária é um tema que tem vindo a ser negligenciado pela medicina, pouco conhecido e menos entendido pela sociedade e pelos profissionais de saúde, sendo que estes dificilmente valorizam as queixas sexuais dos pacientes idosos. Tem-se portanto a ideia de que os profissionais de saúde evitam este assunto por medo de não saberem o que fazer com as perguntas que os utentes Ihes podem colocar. $^{8}$

Assim, se a sociedade evita o assunto, se os próprios idosos têm constrangimento de falar abertamente com os profissionais de saúde sobre o tema e se estes últimos raramente se manifestam em relação a esta temática, o panorama sexual da terceira idade ficará inexoravelmente abandonado ao conformismo e à apatia cultural.

Depois de se conhecer a sexualidade em todas as idades pode-se garantir que a questão da idade é apenas um pequeno e simples detalhe, e o que é visto como problema é a sexualidade, que envolve normas, valores, heranças, atitudes e comportamentos. ${ }^{4}$

\section{Sexo e Demência}

Define-se demência como uma situação adquirida e permanente de défice global das faculdades mentais, que abrange as capacidades cognitivas (incluindo a sensopercepção e a comunicação), afectivas e volitivas, o comportamento e a personalidade. ${ }^{9}$

Na prática, quando se fala em demência tem-se em especial atenção o conjunto de alterações que afectam a esfera cognitiva, por serem essas as perturbações que mais precocemente chamam a atenção dos familiares e dos clínicos. Mas, tarde ou cedo, os outros domínios da vida mental são atingidos, e o funcionamento do organismo no seu todo acaba por ser afectado. ${ }^{5}$

A doença de Alzheimer é causa mais comum de demência, estando presente em cerca de três quartos dos casos. Recentes estimativas sugerem que, em 2000, 18-25 milhões de pessoas eram afectadas por esta patologia, sendo que estes casos podem aumentar para 32-40 milhões de pessoas, até ao ano de 2020. A demência é considerada como a doença da terceira idade, sendo que apenas $2 \%$ da população afectada tem idade inferior a 65 anos. Após os 65 anos, a prevalência da demência tende a dobrar por cada 5 anos. ${ }^{10}$

A intimidade, a sexualidade e os comportamentos sexuais são temas de abordagem ainda tímida em algumas das instituições prestadoras de cuidados ao idoso com défice cognitivo. Da mesma forma que os doentes demenciados experienciam alterações na cognição, memória e julgamento, a expressão da sua sexualidade pode também conhecer modificações e assim colocar problemas de difícil gestão tanto no contexto comunitário como do próprio casal. ${ }^{5}$

Quando se pensa numa pessoa com demência focaliza-se na maioria das vezes, o desempenho cognitivo e a vida quotidiana, colocando de parte as questões relacionadas com a sexualidade destes indivíduos. Uma abordagem centrada na pessoa implica invariavelmente o estudo da perda cognitiva em causa, mas também a recolha de dados biográficos, do estado geral de saúde, da resposta prévia a factores de stress e relativos à história sexual e personalidade pré-mórbida do doente. ${ }^{5}$

Apesar de existir o estereótipo, por parte de cuidadores (formais e informais) e de profissionais de saúde, de que os indivíduos demenciados são seres assexuados, de acordo 
com alguns estudos, tal premissa não se verifica. Existem estudos que relatam que a apatia sexual é documentada em apenas $23 \%$ dos casos estudados, e que $53 \%$ dos doentes de Alzheimer sofrem de disfunção eréctil. ${ }^{11,12}$

A literatura refere-se à demência como um estado que pode legitimar alguma bizarria no comportamento sexual dos doentes, devendo ficar sempre presente que estas pessoas têm uma doença cerebral que causa alterações a nível cognitivo e comportamental. No entanto, são observadas flutuações ao nível do desempenho cognitivo que resultam frequentemente na interrogação quanto ao rótulo de demenciados, postado pelos cuidadores, e na consequente responsabilização de alguns destes utentes, pelos seus demais comportamentos. ${ }^{13}$

Apesar da incontestável importância destas questões, há que lembrar que a maioria dos indivíduos demenciados são também adultos, sendo por isso natural que expressem sentimentos e comportamentos sexuais típicos desta faixa etária, desta forma não podem, de forma alguma ser considerados como seres assexuados ou, no outro extremo, que qualquer comportamento de natureza sexual (mesmo que adequado) seja lido como uma manifestação de hipersexualidade. ${ }^{14}$

Para além de todas as outras questões já levantadas, existe uma fulcral: como reagem os profissionais quando deparados com uma manifestação de comportamento sexual por parte de um dos utentes?

Alguns autores, relataram que 78\% dos técnicos que entrevistaram concordavam que comportamentos como abraçar, dar as mãos ou beijar, aconteciam frequentemente entre os utentes demenciados, havendo em $60 \%$ dos casos a ocorrência de comportamento sexual explícito (envolvendo toque ou tentativa de toque dos órgãos genitais) entre alguns residentes. ${ }^{15}$ Destes comportamentos, o staff reagia com particular desconforto quando a relação ultrapassava a expressão de carinho e assumia contornos eróticos. No entanto, todas as manifestações (entre doentes) despoletaram, nos restantes residentes, conforme o seu grau de lucidez e compreensão, diferentes reacções que variaram desde a indiferença até à zanga e exigência de expulsão do "infractor".

Os problemas sexuais independentes da existência de um parceiro, incluem sobretudo comportamentos inapropriados como despir-se ou masturbar-se em público e o assédio a conhecidos e desconhecidos, com desinibição ao nível da linguagem. ${ }^{5}$

\section{Perspectivas Éticas}

A discussão da sexualidade nos idosos dementes é complexa uma vez que interfere com valores morais, religiosos e culturais.

A demência possui determinadas especificidades que poderão de certa forma, levantar determinados problemas éticos. Em primeiro, o próprio diagnóstico da doença, não sendo exacto, mas cujo peso é relevante, e em segundo o prognóstico daí decorrente, geram a necessidade de dar ao utente a informação a que tem direito. Porém, as dificuldades causadas pela própria patologia, sejam cognitivas e/ou comunicativas, perturbam a forma como esta mesma informação será anunciada. A dependência de terceiros, inevitável no decurso da doença, causa também dificuldades na comunicação do diagnóstico ao próprio utente, uma vez que os cuidadores tendem a tomar decisões pelo próprio utente. Na realidade, todas as características enunciadas dificultam a aplicação dos princípios fundamentais da bioética, nomeadamente o direito ao consentimento informado, à autodeterminação e à tomada de decisões. ${ }^{16}$

Os problemas éticos levantados na sexualidade em idosos dementes variam em função do próprio estado evolutivo da demência.

A intimidade, sexualidade e comportamentos sexuais são temas de abordagem ainda tímida em algumas das instituições prestadoras de cuidados ao idoso com défice cognitivo. Esta realidade é tão mais preocupante quanto maior for a tendência dos cuidadores para pensarem no demenciado não como um adulto dotado de uma identidade sexual, mas como um ser assexuado. Assim enquanto alguns concordam que o doente tem direito à expressão da sua sexualidade (dentro de certos parâmetros) outros podem simplesmente ignorar tal assunto ou até sentir estas manifestações como uma afronta às suas crenças pessoais e códigos morais. ${ }^{5}$

Os profissionais que lidam diariamente com indivíduos com défice cognitivo/demência devem estar conscientes de que à medida que a demência evolui, também as necessidades se modificam, as relações existentes podem sofrer alterações, podem surgir novos relacionamentos e o desejo poderá sofrer flutuações, não alterando, no entanto, o direito que cada adulto tem a permanecer sexualmente activo, desde que assim o deseje e não violente os direitos do outro, independentemente, da idade, capacidade ou preferência sexual. ${ }^{11}$ 
Muitos dos que procuram os serviços de apoio à terceira idade possuem uma convicção: o sexo é apenas para os cognitivamente intactos. No entanto tal facto não se verifica, o que poderá dar origem a situações de difícil resolução. 0 parceiro saudável pode questionar-se se, quando inicia uma interacção sexual, está, ou não, a abusar do outro. A patologia ou a institucionalização do utente podem implicar que o indivíduo "saudável" do casal veja a sua actividade sexual prejudicada e o problema da fidelidade se coloque. ${ }^{5}$

É também comum que um utente demenciado desenvolva novos relacionamentos afectivos quando institucionalizado. Tal situação pode causar problemas de ordem ético moral aos técnicos, que podem não ter formação específica para lidar com estas situações. ${ }^{15}$ Neste tipo de situações, é necessário e importante, que exista um responsável indicado previamente, pelo doente ou cuidador, capaz de interpretar em cada momento os seus desejos. Se a existência deste responsável não for possível, os técnicos deverão agir segundo o princípio da beneficência no interesse do doente, tentando reflectir o juízo que o doente faria por si e para si se estivesse capacitado para tal. ${ }^{14}$

Com a evolução da doença, mais cedo ou mais tarde, sobrevém a dependência psicológica, instrumental e física do doente em relação a terceiros. A autonomia de decisão e a autonomia física vão sendo perdidas. O princípio ético da vulnerabilidade, existente em qualquer doença, torna-se aqui particularmente relevante.

As alterações de comportamento do doente vão surgindo com carácter progressivo ou flutuante e deste modo a limitação da actividade, por meios físicos ou terapêuticos, é uma opção que pode ser necessário tomar em determinadas fases da doença. Do ponto de vista ético, sendo a contenção uma limitação da autonomia pode sim ser tomada, mas só para reduzir prejuízos ou perigos para o doente e só deve ser utilizada neste sentido. ${ }^{16}$

\section{Conclusão}

Após toda uma reflexão sobre sexualidade na terceira idade com especial ênfase em indivíduos demenciados, conclui-se que existe anfibologia em determinados aspectos da demência, da deficiente formação dos técnicos e a envolvência cultural que inibe uma discussão aberta e informada considerando que todas estas questões são promotoras das lacunas existentes na forma como se aborda o tema da sexualidade em adultos demenciados.

Nas instituições de apoio à terceira idade, existentes na actual sociedade, a solução encontrada na gestão de situações ditas embaraçosas passa pela proibição do relacionamento sexual entre os doentes e/ou pela constante interpretação de um acontecimento sexual como não o sendo, negando-o. Assim tanto o staff técnico como o não técnico dão prioridade à protecção do utente e à sua auto-protecção, em simultâneo, perpetuando preconceitos e mitos que reduzem o indivíduo a uma condição assexuada.

Se a sexualidade na terceira idade é dificilmente aceite, esta torna-se ainda mais complexa, particularmente quando associada a problemas do foro da saúde mental. Referindo-se esta como uma área fortemente influenciada pelas atitudes, preconceitos e crenças das entidades cuidadoras, é necessário que estas deliberem esforços no sentido de respeitar os direitos dos pacientes permitindo uma adequada manifestação da sexualidade. No entanto deve ser mantida uma especial atenção, pois algumas destas expressões sexuais podem representar riscos e, consequentemente, necessitar de monitorização e redireccionamento.

Todos os técnicos de saúde deveriam receber formação sobre a saúde sexual na terceira idade e na demência de forma a aprender a estar atentos às necessidades dos pacientes e seus cônjuges, fazendo um esforço no sentido de ultrapassarem as suas próprias inibições acerca do sexo e da sexualidade, principalmente na fase tardia da vida.

0 treino específico para lidar com a expressão sexual destes doentes facilitaria os processos de tomada de decisão, nomeadamente no decidir se as interacções de natureza sexual são permitidas, que tipo de comportamentos sexuais serão aceites, com quem estas interacções poderão acontecer, onde poderão ocorrer e qual o momento e os procedimentos se a intervenção do "staff" se verificar necessária. Finalmente, os técnicos devem manter-se atentos, actualizados e, periodicamente, reavaliar os seus protocolos de intervenção de forma a também nesta área, prestarem a melhor assistência ao doente com demência. 


\section{Referências Bibliográficas}

1. WHO. World Health Organization. Integrating poverty and gender into health programmes: a sourcebook for health professionals: module on ageing. [Online] 2006. [Citado em: Novembro 28, 2010.] Disponível em URL: http://whqlibdoc.who.int/wpro/2006/9290612339_eng.pdf.

2. Custódio, Carla Maria de Faria. Representações e vivências da sexualidade no idoso institucionalizado - Dissertação de mestrado em comunicação em saúde. [ed.] Universidade Aberta. [Online] Maio 2008. [Citado em: Novembro 30, 2010.] Disponível em URL: :http://repositorioaberto. univ-ab.pt/bitstream/10400.2/1230/1/Tese\%20-\%20Reformulada\%20pdf.pdf.

3. WHO. World Health Organization. [Online] World Health Organization. [Citado em: Novembro 30, 2010.] http://www. who.int/en/.

4. Dantas, José Marcos Ribeiro, Silva, Elisangela Martins da and Loures, Marta Carvalho. Socialgest. Lazer e Sexualidade no envelhecimento. [Online] 2004. [Citado em: Outubro 15, 2010.] Disponivel em URL: http://www.socialgest. pt/_dlds/lazersexualidadenosidosos.pdf.

5. Ferros, Lígia Cristina. Sexualidade e Demência. [book auth.] A. Castro-Caldas, A. Mendonça and (Coord.). A Demência de Alzheimer e Outras Demências em Portugal. Lisboa: Lidel, 2005.

6. Azevedo, R. B. Doenças Sexualmente Transmissíveis. São Paulo: Grupo Saúde e Vida, 2002.

7. Fuertes, Antonio and Lopez, Felix. Para comprender la sexualidad. São Paulo: Edições Loyola, 1992.

\section{Conflito de Interesses}

Os autores declaram não ter conflito de interesses em relação a este artigo.

\section{Endereço para Correspondência:}

José Augusto Simões

Email: jars@ua.pt
8. Messina, Mónica. Dimensões do envelhecer na contemporaneidade. Rio de Janeiro: s.n., 2003.

9. Santana, Isabel. A demência de Alzheimer e outras demências - diagnóstico diferencial. A. Castro-Caldas, A. Mendonça and (Coord.). A doença de Alzheimer e outras demências em Portugal. Lisboa: Lidel, 2005.

10. Prince, Martin. Epidemiology of dementia. Psychiatry. 2004, Vols. 3, n¹2.

11. Miller, B., et al. Dietary changes, compulsions and sexual behavior in frontotemporal degeneration. Dementia. 1995, Vol. 6, 195-199.

12. Davies, H., et al. Sexuality and intimacy in Alzheimer's patients and their partners. Sexuality and Disability. 1998, Vols. 6, n³, 193-203.

13. Archibald, C. Sexuality and dementioa in residential care. Sexual and relationship therapy. 2002, Vols. $17, n^{\circ} 3$, 301-309.

14. Hillman, Jennifer L. Clinical perspectives on elderly sexuality.

15. Holmes, D., Reingold, J. and Teresi, J. Sexual expression and dementia: views of caregivers. International Journal of Geriatric Psychiatry. 1997, Vol. 12, pp. 695-701.

16. Pontes, Celso. Sexualidade e demência. Aspectos éticos na Doença de Alzheimer. Lisboa: Lidel, 2005.

17. Kennedy, Gary J., Martinez, Moises M. and Garo, Nicholas. Sex and Mental Health in Old Age. Primary Psychiatry. 2010, Vol. 17 (1).

18 Santana, Isabel. A doença de Alzheimer e outras demências - Diagnóstico diferencial. A. Castro-Caldas, A. Mendonça and (Coord.). A doença de Alzheimere outras demências em Portugal. Lisboa: Lidel, 2005. 